\title{
Inferring the Role of the Metabolism of Polyamines in the Phytopathogenic Bacteria Pseudomonas Syringae: A Meta-Analysis Approach
}

\section{Leandro Solmi}

Laboratorio de Estrés Biótico y Abiótico en Plantas-Instituto Tecnológico de Chascomús (INTECh), Consejo Nacional de Investigaciones Científicas y Técnicas-Universidad Nacional de General San Martín (CONICET-UNSAM), Buenos Aires (CP7130)

\section{Hernan Guillermo Rosli}

Laboratorio de Interacciones Planta Patógeno-Instituto de Fisiología Vegetal (INFIVE)

\section{Marina Alejandra Pombo}

Laboratorio de Interacciones Planta Patógeno-Instituto de Fisiología Vegetal (INFIVE)

\section{Santiago Stalder}

Laboratorio de Estrés Biótico y Abiótico en Plantas-Instituto Tecnológico de Chascomús (INTECh), Consejo Nacional de Investigaciones Científicas y Técnicas-Universidad Nacional de General San Martín (CONICET-UNSAM), Buenos Aires (CP7130)

\section{Franco Rubén Rossi}

Laboratorio de Estrés Biótico y Abiótico en Plantas-Instituto Tecnológico de Chascomús (INTECh), Consejo Nacional de Investigaciones Científicas y Técnicas-Universidad Nacional de General San Martín (CONICET-UNSAM), Buenos Aires (CP7130)

\section{Fernando Matías Romero}

Laboratorio de Estrés Biótico y Abiótico en Plantas-Instituto Tecnológico de Chascomús (INTECh), Consejo Nacional de Investigaciones Científicas y Técnicas-Universidad Nacional de General San Martín (CONICET-UNSAM), Buenos Aires (CP7130)

\section{Oscar Adolfo Ruiz}

Laboratorio de Estrés Biótico y Abiótico en Plantas-Instituto Tecnológico de Chascomús (INTECh), Consejo Nacional de Investigaciones Científicas y Técnicas-Universidad Nacional de General San Martín (CONICET-UNSAM), Buenos Aires (CP7130)

\section{Andrés Gárriz ( $\square$ garriz@intech.gov.ar)}

Laboratorio de Estrés Biótico y Abiótico en Plantas-Instituto Tecnológico de Chascomús (INTECh), Consejo Nacional de Investigaciones Científicas y Técnicas-Universidad Nacional de General San Martín (CONICET-UNSAM), Buenos Aires (CP7130)

\section{Research Article}


Keywords:

Posted Date: January 17th, 2022

DOI: https://doi.org/10.21203/rs.3.rs-1181782/v1

License: (c) (1) This work is licensed under a Creative Commons Attribution 4.0 International License. Read Full License 


\section{Abstract}

Pseudomonas syringae is a phytopathogenic bacteria causing disease in a wide variety of economically important plant species. To succeed in plant invasion, these bacteria rely on virulence mechanisms that subvert plant immunity and create favorable conditions for growth. This process requires a precise regulation in the production of important proteins and metabolites. Among them, the family of compounds known as polyamines have attracted attention as they are involved in essential cellular processes. However, it is not known yet how phytopathogenic bacteria regulates polyamine homeostasis in the plant environment. In this work, we conducted a meta-analysis of publicly available transcriptomic data with the purpose to understand the regulation of the metabolism of polyamines and their links to pathogenicity in $P$. syringae. We demonstrated that polyamine synthesis is induced in the early stages of the disease, along with gene expression activation and energy production. On the contrary, the synthesis of these compounds is repressed whereas its transport is up-regulated at later stages, which correlates with the expression of virulence genes and the metabolism of nitrogen and carboxylic acids. We also showed that plant defense mechanisms partially hinder polyamine synthesis, which could reduce cell fitness in the plant environment. In addition, our analysis suggested that a proper bacterial response to oxidative conditions requires a reduction in polyamine production. The implications of these conclusions are discussed.

\section{Introduction}

Polyamines comprehend a family of biological compounds that are essential for all living organisms. They are positively charged at physiological $\mathrm{pH}$ and bind to polyanionic compounds such as RNA, DNA, proteins and cell membranes ${ }^{1}$. This ability explains their important participation in RNA translation, DNA replication, membrane stability and cell stress tolerance. The most abundant polyamines in bacteria are the diamine putrescine (Put) and the triamine spermidine (Spd) ${ }^{2}$, even though polyamines with chemical similarities such as cadaverine and norspermidine might be also dominant in some species. In turn, the occurrence of the tetraamine spermine (abundant in mammals and plants) is rather rare.

Intracellular and extracellular contents of polyamines are controlled by a tight regulation of their pathways of synthesis, catabolism, and transport (Figure 1). Depending on the initial substrate, two pathways are used for the formation of Put. One of them requires the decarboxylation of the amino acid arginine, whereas an alternative pathway decarboxylates ornithine instead. Decarboxylation of arginine is carried out by SpeA (arginine decarboxylase) and forms agmatine, this metabolite thus being later converted into Put by two consecutive enzymatic steps catalysed by agmatine deiminase (AguA) and $N$ carbamoylputrescine amidohydrolase (AguB). In turn, ornithine is decarboxylated by SpeC (ornithine decarboxylase) to obtain Put as the final product. Then, Put is transformed into Spd by SpeE (spermidine synthase) with the addition of amino propyl groups provided by decarboxylated S-adenosyl methionine, which in turn is the product of the enzymatic step catalysed by SpeD (S-adenosyl methionine decarboxylase). This anabolic pathway is quite conserved in bacteria and several studies demonstrated their significance for growth, stress tolerance and virulence ${ }^{2-9}$. Despite the catabolic and transport 
pathways have been less explored, there is also evidence suggesting that they contribute to polyamine homeostasis and cell fitness ${ }^{10-12}$. Several routes have been described for the degradation of polyamines in bacteria. First, they can be converted into glutamyl-derivatives by the action of glutamyl-polyamine synthases and then oxidized to gamma-amino butyric acid (GABA) via glutamyl-polyamine oxidases. This involves the protein families PauA and PauB (as they are known in Pseudomonas aeruginosa), respectively ${ }^{13}$. Put might also be transaminated by SpuC using 2-oxo-glutaric acid or pyruvate as amino receptors to render gamma-amino butanal, which is then converted to GABA. In addition, the existence of a Spd dehydrogenase protein has been described in some species ${ }^{14,15}$, but this enzyme is not widespread in bacteria. In turn, a combination of transport systems participates in the incorporation as well as the secretion of polyamines, and bacterial genomes generally show redundancy in the cognate genes ${ }^{16-20}$. Among these transporters, PotABCD, PotFGHI, PotE, and SapBCDF are the most studied. PotABCD and PotFGHI are involved in Spd and Put incorporation, respectively, whereas PotE and SapBCDF seem to be involved mostly in polyamine secretion.

The roles played by these pathways in bacteria have been studied in greater extent in Escherichia coli and $P$. aeruginosa, but also in other human pathogens such as Streptococcus pneumoniae, Vibrio cholerae, and Salmonella enterica. We currently count on a rather incomplete notion of the importance of polyamines in growth and virulence of phytopathogenic bacteria, even though some reports revealed they are essential in these organisms as well ${ }^{21}$. In this trend, Spd is required for the synthesis of the toxin Phevamine A in Pseudomonas syringae, which helps to suppress the oxidative burst induced in plant tissues upon recognition of the pathogen ${ }^{22}$. In addition, the secretion of Put by phytopathogenic bacteria has recently attracted some attention. This process was demonstrated to occur in different bacterial species such as Ralstonia solanacearum growing at the plant xylem, $P$. syringae dwelling at the apoplastic compartment, and Dickeya zeae growing under in vitro conditions ${ }^{23-25}$. On the basis of different reports demonstrating that polyamines have the ability to scavenge reactive oxygen species (ROS) and activate the antioxidant machinery ${ }^{26-29}$, it was proposed that Put secretion by bacteria constitutes a mechanism to counteract the oxidative stress imposed by plants at the site of the infection, a function that remains to be corroborated. Alternatively, it was proposed that Put secretion in the $D$. $z e a e /$ maize interaction is involved in cell-to-cell communication during the invasion of the host ${ }^{25}$. More work is required to reach a better understanding of the roles that polyamines play in such microorganisms.

As the number of molecular studies examining phytopathogenic bacteria is constantly growing, metaanalysis of publicly available transcriptomic data represents a wonderful tool to comprehend the incidence of gene networks in pathogenesis ${ }^{30}$. It is plausible that the comparison between these studies will lead to the recognition of common gene expression patterns and the identification of the metabolic pathways implicated in cell behavior. Under this premise, we speculated that this kind of approach could enable us to discern the signatures that characterize the regulation of the metabolism of polyamines in bacterial pathogens of plants and find the associations between the homeostasis of polyamines and bacterial virulence. 
We focused this study on transcriptomic data obtained from the bacterial species $P$. syringae. This plant pathogen is worldwide distributed and more than 50 pathovars have been described, which altogether cause disease in the most important crops ${ }^{31,32}$. We also contemplated other plant and human pathogens to test whether the identified expression signatures could be extrapolated to non-related pathogenic systems. Our work demonstrates that polyamine synthesis is induced initially during plant colonization, a phenomenon that can be partially suppressed by plant defense responses. In addition, even though the expression of genes from the catabolism and transport of polyamines varied among studies, we were still able to conclude that polyamine incorporation is induced in later stages of infection, probably as an attempt to maintain the supply of these important metabolites when their synthesis is reduced. Finally, an interesting finding was that polyamine synthesis is repressed under oxidative stress, which disagrees with the accepted idea that polyamines are required for tolerance to these conditions. The implications of such findings are discussed.

\section{Results}

Bacteria repress polyamine metabolism under apoplastic-like conditions. As a first approach to evaluate the relationship between the homeostasis of polyamines and bacterial virulence, we looked for works comparing the transcriptome of cells growing in hrp-inducing medium (HIM) and rich media. HIM composition resembles nutritional conditions of plant apoplast such as low $\mathrm{pH}$ and reduced nitrogen/carbon ratio and consequently induces the expression of the $h r p / h r c$ genes. The $h r p / h r c$ cluster encodes type 3 secretion system (T3SS) components translocating effector proteins into plant cells and are essential for pathogenesis ${ }^{33,34}$. Two works following these criteria were found. Vandelle et al ${ }^{35}$ used a multistrain-whole genome platform to evaluate early responses in three biovars of $P$. syringae $\mathrm{pv}$. actinidiae (Psa 1, 2 and 3) as well as in P. syringae pv. tomato DC3000 (Pst), and Nobori et al ${ }^{36}$ examined the transcriptional profile of Pst by RNAseq. Figure 2 shows the expression levels of genes involved in synthesis, catabolism, and transport of polyamines when cells grow in HIM compared to rich media. This analysis evidenced the down-regulation of the anabolic pathway, even though the extension of the repression as well as the genes being repressed varied among species and biovars. It is worthy to note that either the expression of $s p e E$ or $s p e D$ was diminished, suggesting that the synthesis of Spd (and seemingly not of Put) is blocked in response to apoplast-mimicking conditions. In turn, relatively minor changes in the expression of genes involved in the degradation and transport of polyamines were evidenced. In this trend, down-regulation of polyamine catabolism and transport was more evident in Psa3(2) as well as in Pst, which agrees with the similarities between these strains reported by Vandelle et $a^{35}$ in general gene expression. Interestingly, Psa3 biovars strongly up-regulate the T3SS genes compared to other biovars and are responsible for severe outbreaks of bacterial canker in kiwi fruits in important production areas ${ }^{35}$. Whether the repression of the polyamine metabolism is associated to the formation of T3SS and aggressiveness of plant pathogens should be studied in further research.

Polyamine metabolism is induced in bacteria during plant invasion. We then expanded our investigation by analysing transcriptomic profiles in Pseudomonas species during their growth in plant tissues. For this 
purpose, five studies using $P$. syringae pathovars were selected. Lovelace et $a^{37}$ and Nobori et al ${ }^{36,38}$ evaluated gene expression in Pst invading Arabidopsis, whereas Yu et al ${ }^{39}$ and McAtte et al ${ }^{40}$ focused their research in P. syringae pv. syringae B728a (PSs) and Psa colonizing Phaseolus vulgaris and Kiwifruit, respectively. It is important to note that the basal conditions to which all samples were compared in these works were different. Thus, whereas Lovelacce et al ${ }^{37}$ and Nobori et al ${ }^{36,38}$ used cells grown in the rich medium King's $B$ and McAtte et al ${ }^{40}$ used the rich medium LB, Yu et al ${ }^{39}$ on the contrary considered bacteria cultivated in minimal nutrient conditions. Therefore, in the light of the observations described in the previous section, the changes in gene expression caused by minimal media should be considered to interpret the results correctly.

In contrast to the expression profiles observed during growth under in vitro conditions, this analysis demonstrates an early induction of polyamine biosynthesis in Pst (during the first $6 \mathrm{~h}$ after the inoculation, 6 hai) (Figure 3A). Thus, genes in charge of the synthesis of Put (SpeA, SpeC, AguA and $A g u B$ ) and Spd (SpeD, SpeE) resulted up-regulated. Interestingly, genes related to Put synthesis were not differentially expressed later at 48 hai and the synthesis of Spd was suppressed in both Pst and Pss. Therefore, we speculate that a rapid induction of polyamine synthesis is important at the start of plant invasion in these strains. Besides, either the repression of this pathway is required later during the development of disease or rather it is provoked by the activation of the plant defense system. In turn, the analysis of expression of genes from the catabolism and transport of polyamines under in planta conditions is rather inconclusive, as many of them showed contrasting expression patterns among the dataset included. The only consistent observation was an early repression of spuHand induction of potD1. It could be plausible that these pathways are more sensitive to different experimental setups and/or that their regulation strongly depends on the bacterial genotype. In fact, we observed that expression profiles shown by Psa differs considerable from those observed in other strains, as polyamine biosynthesis was not regulated in the early period of plant invasion, and most of the genes involved in polyamine catabolism and transport were induced throughout this process.

In the interest of confirming if polyamine synthesis is important during early plant invasion stages, we searched for phenotypes in mutants of the cognate genes at the Fitness Browser database described at Helmann et al ${ }^{41,42}$ (https://fit.genomics.Ibl.gov/cgi-bin/myFrontPage.cgi). In agreement with this, we found that the transposon-mediated disruption of the speE gene in Pss leads to a mild negative fitness score when growing in the apoplast of green beans (data not shown), suggesting that at least at the start of pathogenesis the anabolism of Spd results important.

Two main defense responses are triggered in plants against the attack of potential pathogens ${ }^{43}$. The first layer of defense is activated upon the recognition of pathogen associated molecular patterns (PAMPs, such as the flagellin peptide flg22 and chitin) and is known as PTI (PAMP-triggered immunity). Pathogens introduce effectors inside plant cells to counteract PTI and promote virulence ${ }^{44}$. Resistant plants can detect directly or indirectly some of these effectors initiating a second defense response, which has been coined as Effector-triggered immunity (ETI) or nucleotide-binding Leu-rich repeat (NLR)- 
triggered immunity (NTI) ${ }^{45}$. Our analysis showed that the pre-induction of PTI (by treating plants with flg22 before inoculation) hinders the induction of polyamine biosynthetic genes and leads to the upregulation of genes from the transport systems SapBCDF and PotABCD (Figure 3B, flg22 vs. mock). In agreement with this observation, the efectorless polymutant $P$ stD $36 \mathrm{E}$ (unable to supress plant immune system) demonstrated a relatively minor induction of polyamine biosynthesis compared to WT Pst, whereas components of the $\operatorname{sap} B C D F / p o t A B C D$ operons were also induced. These results suggest that plant defense mechanisms affect polyamine synthesis in bacteria, which conduces to the activation of polyamine incorporation to overcome their limitation. In addition to that, our analysis also indicates that the translocation of bacterial effectors helps to counteract the negative effects of plant defense responses on polyamine homeostasis. In turn, the activation of ETI (evaluated by plant inoculation with strains expressing either the avirulence genes avrRPT2 or avrRps4) originate slight effects on bacterial polyamine metabolism, as just a higher induction of genes coding for polyamine transporters in the PstAvrRpt2 strain were verified. It should be considered though that ETI causes a rapid induction of plant defense mechanisms that strongly reduces pathogen's growth. Thus, it is possible that the effects on bacterial polyamine metabolism could be masked on such drastic environmental conditions.

\section{Different networks are coordinated with polyamine homeostasis during plant invasion. The broad}

diversity of conditions used in the datasets included in this study enabled us to perform a co-expression analysis with the purpose of identifying gene modules being co-ordinated with the homeostasis of polyamines. We first analysed the data collected by Nobori et al ${ }^{36,38}$ using Pst/Arabidopsis as a model system. In this case, four genes coding for polyamine biosynthetic enzymes ( $s p e A, s p e C$, speE and speD), one for a transporter (potF) and four involved in polyamine catabolism (pauA1, pauA2, pauA4 and spuC) were found to establish significant co-expression relationships with other gene nodes. An evaluation of these groups of genes as well as GO term enrichment analysis identified over-representation of gene categories intimately related to nitrogen compound biosynthesis, carboxylic acid metabolism, energy generation, primary metabolic processes, transmembrane transport, gene expression and macromolecular biosynthetic processes (Figure 4A and 4C). Interestingly, genes from different polyamine pathways established contrasting relationships with these nodes. Thus, whereas polyamine anabolic genes showed positive co-expression scores with membrane transport, amino acid synthesis and regulation of gene transcription/translation, those in charge of polyamine catabolism showed negative scores, suggesting the existence of regulatory mechanisms that avoid simultaneous activation of both pathways. It is interesting to note also that polyamine biosynthesis shows positive correlation with genes involved in energy generation (ATP synthase subunits and electron transport proteins) but negative correlation with genes involved in flagellum formation (Figure 4A). This indicates that energy generation is strongly associated with polyamine synthesis, and that polyamines are not required (or they might be even counter-productive) for the activation of bacterial movement (Figure 4A). We then conducted a dendrogram clustering analysis to better understand the links in the regulation of these gene groups. Thus, the identified biosynthetic genes are grouped together in cluster III (Figure S1A), which includes genes induced at the beginning of plant invasion ( 6 hai) and repressed at later stages (48 hai). Interestingly, these genes are also repressed in samples taken from PTI-induced plants or in the 
hypovirulent strain Pst-D36E, as well as in in vitro experiments using both rich or minimal media. In turn, the polyamine catabolic genes were ordered in cluster II. Even though this group is also mildly induced in the early periods of plant invasion and repressed in vitro, the outstanding feature of these genes is a strong induction at later stages of the infection. Altogether, these results agree with our previous assumption that polyamine biosynthesis is required to ensure bacterial fitness at the start of plant invasion, which would be related to the effects of polyamines on gene expression regulation and energy production. Polyamine accumulation, on the contrary, would not be required at subsequent stages, where the catabolic pathway is activated to reduce their contents.

We then performed the same analysis using the dataset published by Yu et al ${ }^{39}$. This work compared gene expression in the strain Pss B728a during the infection of $P$. vulgaris 48 hai (apoplastic and epiphytic growth) and growth under different stressful conditions in vitro (Figure 4B). A much broader list of genes from the different pathways of the metabolism of polyamines was identified showing significant co-expression scores. GO term enrichment analysis indicated that categories belonging to degradative pathways and membrane transport were over-represented (Figure 4D). Interestingly, even though most of these genes were linked to others through positive correlations, they were clustered in different groups (Figure S1B) and many of these clusters included genes from different polyamine metabolism pathways. Given that only samples taken 48 hai were considered in this dataset, it can be hypothesised that at this stage of infection precise regulatory mechanisms modulates specific genes from the different pathways of the polyamine metabolism rather than regulating the entire route. Importantly, these regulatory mechanisms also operate in response to stressful conditions. The biosynthetic gene speA is mostly linked by negative edges to genes from secondary metabolism and regulators of gene transcription and translation (Figure 4B). This observation suggests that, as concluded above, polyamine synthesis may not be relevant for gene expression as it is at the start of the process. speA was grouped in cluster I, genes that are mildly repressed in response to low nitrogen availability but remained unaltered under other growing conditions (Figure S1B). Thus, it is conceivable that bacteria face a reduction in nitrogen through the development of the disease and respond by repressing the expression of speA to limit the use of nitrogen in favour of the synthesis of other essential compounds. Cluster II contains the operon formed by the Put/Spd transport system PotFGHI, the Put exporter PotE(1), the catabolic oxidase PauB3 and the Spd synthase SpeE. These genes established positive corelations with gene expression regulators and transmembrane proteins, which shows a mild induction when cells are grown under osmotic conditions but not in planta, suggesting that they play a role in this type of stress by result dispensable in the later stages of plant colonization. In turn, cluster III includes the largest amount of polyamine metabolism genes. All these genes belong to the catabolic and transport pathways and establish positive correlation with a large amount of genes up-regulated under low $\mathrm{N}$ conditions. It agrees with the idea that the induction of polyamine catabolism and transport helps to optimize the use of nitrogen under such stressful conditions. Interestingly, these genes also showed positive edges with many genes that conform the hrp/hrc operons, indicating that their induction in response to low $\mathrm{N}$ contents accompanies the activation of bacterial virulence. At last, cluster IV grouped genes up-regulated in planta, but not under other stressful conditions. In this cluster, we identified genes belonging to the 
transporter systems PotABCD and PotFGHI, confirming the idea that transport of polyamines might play an important role during later periods of the infection.

\section{Polyamine metabolism is shut-down during the activation of the anti-oxidative response. Polyamines} have been described as essential metabolites to cope with oxidative stress in bacteria ${ }^{46-50}$. However, our co-expression analysis shown in the previous section did not show a significant correlation with genes involved in redox homeostasis. The only exception is a positive correlation between the gene coding for the catalase KatG and the glutamyl-Put synthase PauA2 in Figure 4A. Interestingly, it was demonstrated that KatG (along with KatB) is required for full virulence in $P s t^{51}$ and the induction of this gene in response to $\mathrm{H}_{2} \mathrm{O}_{2}$ in $\mathrm{E}$. coli is dependent on polyamines ${ }^{50}$. Therefore, we wondered if the activation of the detoxifying machinery in bacteria growing under oxidative stress is accompanied by modifications in the metabolism of polyamines. With this in view, we looked first for transcriptomic data studying bacterial gene expression in response to $\mathrm{H}_{2} \mathrm{O}_{2}$, and selected works that stressed cells for a short period to evaluate the immediate response. In this case, we also included species from other genera to test the existence of widespread regulatory mechanisms. As shown in Figure 5, our analysis that the metabolism of polyamines remains almost unaltered in response to stress, except for the repression of Put or Spd synthesis shortly after the addition of $\mathrm{H}_{2} \mathrm{O}_{2}$ to the culture media. A stronger repression of polyamine synthesis and transport occurs in E. coli and S. enterica (Figure S2), suggesting that in these cases an earlier regulation of the intracellular concentration of these metabolites might be crucial for the antioxidant response. In fact, oxidative stress hallmark genes such as oxy $R$, soxS and sox $R$ resulted more highly activated than in Pseudomonads at the time points analysed. Even so, we conceive that despite suitable levels of polyamines might be required to activate essential component of the antioxidant machinery as reported previously, a rapid repression of polyamine metabolism occurs as part of the stress response. It would be interesting to explore, then, how phytopathogenic bacteria adapt the metabolism of polyamines to cope with the oxidative stress imposed by plants without affecting cell fitness and pathogenesis.

\section{Discussion}

The growing number of publicly available transcriptomic datasets represents a powerful source for the identification of the main features involved in particular cellular processes, such as growth, development, and stress resistance ${ }^{30}$. In the case of pathogenic microbes, these studies may provide a good deal of information concerning common mechanisms participating in the activation of virulence, and at the same time, they offer the possibility to evaluate the relative importance of specific pathways. In this work, we explored the importance of the metabolic routes maintaining the homeostasis of polyamines for pathogenesis and oxidative stress response in bacterial species from the genera Pseudomonas, with an emphasis in P. syringae.

We first compared gene expression levels in cells growing in minimal media in relation to rich media. Minimal media are thought to mimic apoplastic conditions, mainly low $\mathrm{N} / \mathrm{C}$ ratio and acidic $\mathrm{pH}$, which 
lead to the induction of virulence-associated $h r p / h r c$ operons coding for the T3SS ${ }^{33}$. The studies selected for this work offered a time-lapse analysis spanning $8 \mathrm{~h}$ of culture and indicate the existence of a regulatory network suppressing polyamine homeostasis under minimal nutrient environments. These results curtail the importance of polyamines to support growth in minimal media (so that the synthesis of other metabolites is preferred) and besides, suggest that the mechanisms governing polyamine homeostasis differ from those inducing bacterial virulence. Even though this observation contradicts our previous gene expression analysis in Pst using qRT-PCR (where the polyamine biosynthetic gene speC and the catabolic enzymes pauA3 and pauB2 resulted mildly up-regulated in minimal medium), it should be considered that only samples taken 6 hai were analysed in that work and that variations in gene expression before or after that time could have been missed ${ }^{24}$.

A point to be considered is that the plant apoplast is richer in nutrients and that it is sufficient to support the growth of pathogens, at least throughout the early stages of infection ${ }^{52}$. This could explain why in planta growth generates much broader modifications of bacterial transcriptomes than in vitro conditions, which not always mirrors to those observed in in vitro experiments ${ }^{35}$. Thus, expression data from in planta studies should reflect the modulation of the polyamine metabolism and its link with pathogenesis in a more accurate manner. Different studies evaluating the transcriptional profiles of Pseudomonas species in planta were selected to examine the regulation of polyamine pathways during plant colonization. In this trend, our analysis found substantial changes with respect to the metabolic behaviour showed by cells in vitro, as the synthesis of polyamines was induced during the first stages of infection (up to 6 hai) and repressed later with the advance of pathogenesis (48 hai). At the earliest periods considered in this work, it is conceived that plants have already recognized the nature of the pathogen and triggered the first line of defense, whereas the microbe in turn is deploying virulence strategies to resist them. Thus, we concluded that an initial rise in the contents of polyamines via de novo synthesis is required to support cell fitness at this stage. If this is true, mutant strains perturbed in polyamine synthesis should be affected in their virulence. Even though this kind of mutants have not been deeply tested in Pseudomonads yet, a genome-wide fitness profiling made on Pss showed that deletion of $\Delta s p e E$ conduces to mild negative fitness scores when growing in green pepper ${ }^{41}$. The fact that no other mutant strains affected in genes contributing to polyamine biosynthesis or transport show negative scores could be explained on the bases of the existence of redundancy in these pathways. Thus, this would assure the supply of these metabolites during first steps of plant invasion even if these pathways are partially affected by the plant defense systems. In this trend, works in Ralstonia solanacearum (which exclusively depends on the SpeC pathway for Put synthesis) demonstrated that a $\Delta$ speC mutant is hypovirulent in tomato ${ }^{23}$.

The importance of maintaining polyamine contents early in the interaction can be explained through the participation of these compounds in essential molecular mechanisms. For instance, it has been shown that polyamines are required to assure correct ribosome ensemble and general transcription efficiency 53 . In relation to this, our co-expression analysis based on the work made by Nobori et al ${ }^{36,38}$, where most of the samples correspond to cells growing for $6 \mathrm{~h}$ both in vitro and in planta, indicated that genes involved 
in polyamine biosynthesis were coexpressed with ribosome components as well as regulators of transcription/translation. On the contrary, polyamine catabolic genes showed a negative coexpression relationship with the same group of genes. We also distinguished the same pattern of coexpression with genes involved in ATP formation, carboxylic acid and nitrogen metabolism. Thus, polyamine accumulation would be associated to the synthesis of important compounds and a higher rate of energy generation and gene expression. An interesting observation derived from this analysis is the fact that polyamine biosynthetic genes were negatively correlated with genes participating in the formation of the flagella, which suggests that bacterial movement could be affected by polyamines in Pseudomonads. In agreement with this, it was recently demonstrated that Put accumulation by disrupting Spd formation or the Put aminotransferase pathway promotes the transition towards the formation of sessile biofilms in $P$. aeruginosa ${ }^{54}$. However, the link between polyamines and cell movement seems to depend on the bacterial species and the polyamine involved. In this trend, even though deletion of $s p e A$ and $s p e C$ in Yersinia pestis also perturbs biofilm formation ${ }^{55}$, the lack of $s p e A$ in Dickeya zeae and Proteus mirabilis decreases swimming and swarming mobilities ${ }^{7,25}$. It would be interesting to corroborate the significance of polyamine contents for cell movement in $P$. syringae.

We also demonstrated that pre-induction of plant defense responses leads to a minor induction of polyamine biosynthesis and to the up-regulation of polyamine transport genes. This also occurs when the effectorless D36E mutant strain is analysed, emphasising the importance of the T3SS function in avoiding the effects of plant defense mechanisms on pathogen's metabolism. We speculate that a rise in the expression of polyamine transporters under these conditions is a consequence of lower expression of the anabolic genes. The mechanisms underlying the reduction in the synthesis of polyamines could be either 1) a bacterial response to the deleterious environment imposed by the plant defense system, or 2) that plant defense responses directly target the synthesis of these compounds to affect cell fitness. In relation to this, it is known that one of the main defense mechanisms deployed by plants against endophytic bacteria is the accumulation of reactive oxygen species at the apoplast ${ }^{39,52}$, provoking oxidative stress conditions to bacteria dwelling at this niche. With this is mind, we explored transcription datasets obtained from different bacterial species exposed to oxidative stress and found that, even though at different extents, polyamine biosynthetic genes were down-regulated in bacteria. Hence, the reduction in polyamine synthesis might be associated with the induction of the antioxidative machinery that helps to cope with plant defense responses. This seems to contradict the accepted paradigm that polyamines help to cope with this stress ${ }^{11,48,56,57}$. In this trend, secretion of polyamines by $R$. solanacearum at the tomato xylem was proposed by Lowe-Power et al ${ }^{23}$ as a possible mechanism to subdue oxidative stress. These authors demonstrated that Put is accumulated at this compartment presumably as a product of the activation of bacterial metabolism, and that a $\Delta s p e C$ mutant resulted completely avirulent. In relation to this, we described in a previous work that Pst can also secrete large amounts of Put when growing in apoplastic washing fluids, although we could not find a link between this phenomenon and bacterial virulence. Our next experiments will try to use Pst mutants unable to synthesize polyamines to further test their roles in stress tolerance and its link with pathogenesis. 
As mentioned above, our study showed that bacterial polyamine biosynthesis is repressed 48 hai, which is also associated to the induction of polyamine transporters and catabolic genes. A similar scenario is observed in later stages of pathogenic interactions established by other bacterial species. For instance, Xanthomonas axonopodis pv. glycines reduces the expression of $s p e D$ but up-regulates other polyamine biosynthetic and transport genes after 72 hai in soybean ${ }^{58}$, whereas polyamine synthesis was repressed in Xanthomonas oryzae pv. oryzae after 5 days while polyamine transport was activated ${ }^{59}$ (data not shown). Importantly, hierarchical clustering of co-expressed gene analysis demonstrated that transcription profiles from samples taken 48 hai are grouped with those obtained from cells growing in vitro under low nitrogen conditions or in minimal medium, as well as in experiments using primed plants (pre-infiltrated with flg22) or the D36E strain. Based in this, it is reasonable to conclude that bacteria are facing more restrictive conditions at this stage. Besides, the induction of some of the polyamine metabolic genes at 48 hai is correlated with the induction of the T3SS. This observation supports the idea that the modulation of specific genes from the metabolism of polyamines, but not all, would be coordinated with the induction of virulence mechanisms. More work is needed to understand the regulatory mechanisms acting regulating genes from the polyamine metabolism throughout the infection and their impact on pathogenesis.

Altogether, these findings contribute to the hypothesis that polyamine synthesis in phytopathogenic Pseudomonas species is important during the early periods of plant invasion, which is accompanied by the activation of nitrogen and carboxylic acid metabolism, general gene expression and energy generation. Afterwards, this pathway is repressed in later stages with the consequent induction of the transport systems (Figure 6), coordinated with the expression of virulence genes and degradative enzymes. This metabolic shift enables the survival of cells in the plant environment. Whether this behaviour could be extrapolated to other bacterial species should be evaluated with the generation of specific mutant strains perturbed in the different branches of the polyamine metabolism.

\section{Materials And Methods}

Identification of orthologs of the polyamine metabolism genes in bacterial genomes. To identify groups of orthologs in the different bacterial species considered in this work, we followed two approaches. Thus, we explored the Ortholog Group Member database at Pseudomonas Genome DB (httpps://pseudomonas.com), or alternatively, applied the reciprocal best hit method using the NCBI's blastp software ${ }^{60}$. In those cases where this pipeline failed in finding potential orthologs in a defined database, we still included in our analysis the best hit if the query coverage was higher than $80 \%$ and $\mathrm{E}$ value was below $1 \times 10^{-10}$. A list of the genes considered for each strain is shown in Table S1.

Selection of transcriptomic datasets from the public domain. Microarray and RNAseq data were downloaded from the NCBI Gene Expression Omnibus (GEO). GEO repository was queried using keywords such as "Pseudomonas", "Phytopathogenic bacteria", "pathogenic bacteria", "oxidative stress", and "plant infection". In total, nine analyses were selected. Seven of these works were performed in three different pathovars of $P$. syringae and other Pseudomonads such as $P$. putida and $P$. aeruginosa, whereas two 
considered the human pathogens E. coli and S. enterica (see Table 1 for a brief description). We explored data obtained from $P$. syringae growing under in vitro and in planta conditions to unravel the links between polyamine metabolism and pathogenesis, whereas those works evaluating bacterial responses to $\mathrm{H}_{2} \mathrm{O}_{2}$ were used to corroborate the potential role of these metabolites in the stress response. In this last case, we excluded studies analyzing gene expression beyond $1 \mathrm{~h}$ of treatment, as we were interested in the early response. In addition, works that utilize concentrations of $\mathrm{H}_{2} \mathrm{O}_{2}$ higher than $15 \mathrm{mM}$ were also ruled-out as these concentrations are significantly higher than the physiological amounts faced by bacteria in plant tissues. Because of the experimental heterogeneity associated to these datasets and the complexity underlying their normalization, we did not re-analyze them using a unified pipeline, but rather attempted to identify transcriptional patterns in a broader sense by comparing the expression levels reported by the authors in each case. Thus, gene expression levels were obtained from the DEGs (Differentially Expressed Genes) listed in each study, and genes were included in our analysis if the fold change $\left(\log _{2} \mathrm{FC}\right)$ resulted $>|0.5|$ and showed $p$-adjusted values $<0.05$. 
Table 1

Description of the datasets used for the meta-analysis in this work. Bacterial species/strains, Plant host and Growth conditions are listed

\begin{tabular}{|c|c|c|c|c|}
\hline Authors & $\begin{array}{l}\text { Study } \\
\text { accession }\end{array}$ & Bacterial strain & Plant host & Growth conditions \\
\hline \multirow{2}{*}{$\begin{array}{l}\text { Nobori et } \\
\text { al }^{36,38}\end{array}$} & GSE103442 & \multirow{2}{*}{$\begin{array}{l}\text { P. syringae pv. } \\
\text { tomato DC3000 }\end{array}$} & \multirow{2}{*}{$\begin{array}{l}\text { Arabidopsis } \\
\text { thaliana Col-0 }\end{array}$} & \multirow{2}{*}{$\begin{array}{l}\text { In vitro (minimal and } \\
\text { rich media) and In } \\
\text { planta }\end{array}$} \\
\hline & GSE138901 & & & \\
\hline Yu et al ${ }^{39}$ & GSE42544 & $\begin{array}{l}\text { P. syringae pv. } \\
\text { syringae } \mathrm{B} 728 \mathrm{a}\end{array}$ & $\begin{array}{l}\text { Phaseolus } \\
\text { vulgaris cultivar } \\
\text { Bush Blue Lake } \\
274\end{array}$ & $\begin{array}{l}\text { In vitro (minimal and } \\
\text { rich media, stressful } \\
\text { conditions) and In } \\
\text { planta }\end{array}$ \\
\hline $\begin{array}{l}\text { McAtte et } \\
\text { al }\end{array}$ & PRJNA472664 & $\begin{array}{l}\text { P. syringae pv. } \\
\text { actinidiae ICMP } \\
18884\end{array}$ & $\begin{array}{l}\text { Actinidia } \\
\text { chinensis Planch. } \\
\text { var. chinensis } \\
\text { "Hort16A" }\end{array}$ & In planta \\
\hline $\begin{array}{l}\text { Vandelle } \\
\text { et al }{ }^{35}\end{array}$ & GSE164472 & $\begin{array}{l}\text { P. syringae pv. } \\
\text { actinidiae biovars } 1, \\
2 \text { and } 3\end{array}$ & None & $\begin{array}{l}\text { In vitro (minimal and } \\
\text { rich media) }\end{array}$ \\
\hline $\begin{array}{l}\text { Bojanovic } \\
\text { et al } 64\end{array}$ & GSE85475 & P. putida KT2440 & None & $\begin{array}{l}\text { In vitro }\left(\mathrm{H}_{2} \mathrm{O}_{2} \text { amended }\right. \\
\text { media) }\end{array}$ \\
\hline $\begin{array}{l}\text { Jozefczuk } \\
\text { et al } 65\end{array}$ & GSE20305 & E. coli MG1655 & None & $\begin{array}{l}\text { In vitro }\left(\mathrm{H}_{2} \mathrm{O}_{2} \text { amended }\right. \\
\text { media) }\end{array}$ \\
\hline $\begin{array}{l}\text { Chang et } \\
\text { al } 66\end{array}$ & GSE3090 & P. aeruginosa $\mathrm{PA} 01$ & None & $\begin{array}{l}\text { In vitro }\left(\mathrm{H}_{2} \mathrm{O}_{2} \text { amended }\right. \\
\text { media) }\end{array}$ \\
\hline $\begin{array}{l}\text { Liu et al } \\
67\end{array}$ & GSE 155479 & $\begin{array}{l}\text { S. enterica subs. } \\
\text { enterica serovar } \\
\text { Enteritidis ATCC } \\
13076\end{array}$ & None & $\begin{array}{l}\text { In vitro }\left(\mathrm{H}_{2} \mathrm{O}_{2} \text { amended }\right. \\
\text { media) }\end{array}$ \\
\hline
\end{tabular}

\section{Co-expression analysis.}

Datasets uploaded by Nobori et al ${ }^{36,38}$ and Yu et al ${ }^{39}$ were used to calculate Pair-wise gene expression Pearson correlation across all the samples to generate a similarity matrix, which then were the inputs for the construction of co-expression networks (using R/WGCNA version 1.34) as described by Sharma et al 61 . Hierarchical cluster trees were created by setting gene co-expression module seizes of 30, deepSplit at level 1 and tree mergecutHeight at 0.20 . Figures were created using Cytoscape ${ }^{62}$ and enriched GO terms were identified using BiNGO plugin ${ }^{63}$.

\section{Declarations}




\section{Acknowledgments}

L. Solmi, H. G. Rosli, M. A. Pombo, F. R. Rossi, F. M. Romero, O. A. Ruiz and A. Gárriz, are members of the Research Staff of the Consejo Nacional de Investigaciones Científicas y Técnica s (CONICET, Argentina). This work was partly funded by the project "Universidades Agregando Valor" \#8550 from the Secretaría de Políticas Universitarias, Argentina. The authors would like to thank Josefina Gárriz for his diligent proofreading of this manuscript.

\section{Author Contributions}

L.S and A.G. designed the study; S.S., L.S. and A.G. prepared figures; A.G. wrote the main text. All authors analyzed and reviewed the manuscript.

\section{Competing interests}

The author declares no competing interests.

\section{References}

1. Michael, A. J. Polyamines in Eukaryotes, Bacteria, and Archaea. J Biol Chem 291, 14896-14903, doi:10.1074/jbc.R116.734780 (2016).

2. Gevrekci, A. O. The roles of polyamines in microorganisms. World journal of microbiology \& biotechnology 33, 204, doi:10.1007/s11274-017-2370-y (2017).

3. Guerra, P. R. et al. Polyamine depletion has global effects on stress and virulence gene expression and affects HilA translation in Salmonella enterica serovar typhimurium. Research in Microbiology 171, 143-152, doi:https://doi.org/10.1016/j.resmic.2019.12.001 (2020).

4. Chagneau, C. V. et al. The Polyamine Spermidine Modulates the Production of the Bacterial Genotoxin Colibactin. mSphere 4, e00414-00419, doi:10.1128/mSphere.00414-19 (2019).

5. Igarashi, K. \& Kashiwagi, K. Effects of polyamines on protein synthesis and growth of Escherichia coli. J Biol Chem 293, 18702-18709, doi:10.1074/jbc.TM118.003465 (2018).

6. Banerji, R., Kanojiya, P., Patil, A. \& Saroj, S. D. Polyamines in the virulence of bacterial pathogens of respiratory tract. Molecular oral microbiology 36, 1-11, doi:10.1111/omi.12315 (2021).

7. Shah, P. \& Swiatlo, E. A multifaceted role for polyamines in bacterial pathogens. Mol Microbio/ 68, 416, doi:MMI6126 [pii] 10.1111/j.1365-2958.2008.06126.x (2008).

8. Banerji, R., Kanojiya, P. \& Saroj, S. D. Role of interspecies bacterial communication in the virulence of pathogenic bacteria. Crit Rev Microbio/ 46, 136-146, doi:10.1080/1040841x.2020.1735991 (2020).

9. Nanduri, B. \& Swiatlo, E. The expansive effects of polyamines on the metabolism and virulence of Streptococcus pneumoniae. Pneumonia 13, 4, doi:10.1186/s41479-021-00082-x (2021).

10. Bolard, A. et al. Production of Norspermidine Contributes to Aminoglycoside Resistance in pmrAB Mutants of Pseudomonas aeruginosa. Antimicrob Agents Chemother 63, doi:10.1128/aac.01044-19 
(2019).

11. Johnson, L., Mulcahy, H., Kanevets, U., Shi, Y. \& Lewenza, S. Surface-localized spermidine protects the Pseudomonas aeruginosa outer membrane from antibiotic treatment and oxidative stress. $J$ Bacteriol 194, 813-826, doi:10.1128/JB.05230-11 (2012).

12. Liu, W. et al. Functional characterization of murB-potABCD operon for polyamine uptake and peptidoglycan synthesis in Streptococcus suis. Microbiol Res 207, 177-187, doi:10.1016/j.micres.2017.11.008 (2018).

13. Schneider, J. \& Wendisch, V. F. Biotechnological production of polyamines by Bacteria: recent achievements and future perspectives. App/ Microbiol Biotechnol, doi:10.1007/s00253-011-3252-0 (2011).

14. Dasu, V. V., Nakada, Y., Ohnishi-Kameyama, M., Kimura, K. \& Itoh, Y. Characterization and a role of Pseudomonas aeruginosa spermidine dehydrogenase in polyamine catabolism. Microbiology 152, 2265-2272, doi:152/8/2265 [pii] 10.1099/mic.0.28920-0 (2006).

15. Hisano, T., Murata K, Kimura A, Matsushita K, Toyama H, Adachi O. Characterization of membranebound spermidine deshydrogenase of Citrobacter freundii. Biosci Biotechnol Biochem. 56, 1916-1920 (1992).

16. Shah, P., Romero, D. G. \& Swiatlo, E. Role of polyamine transport in Streptococcus pneumoniae response to physiological stress and murine septicemia. Microb Pathog 45, 167-172, doi:S08824010(08)00066-1 [pii] 10.1016/j.micpath.2008.05.001 (2008).

17. Shah, P., Nanduri, B., Swiatlo, E., Ma, Y. \& Pendarvis, K. Polyamine biosynthesis and transport mechanisms are crucial for fitness and pathogenesis of Streptococcus pneumoniae. Microbiology 157, 504-515, doi:mic.0.042564-0 [pii] 10.1099/mic.0.042564-0 (2011).

18. Schiller, D., Kruse, D., Kneifel, H., Kramer, R. \& Burkovski, A. Polyamine transport and role of potE in response to osmotic stress in Escherichia coli. J Bacterio/ 182, 6247-6249 (2000).

19. Pipkins, H. R., Bradshaw, J. L., Keller, L. E., Swiatlo, E. \& McDaniel, L. S. Polyamine transporter potABCD is required for virulence of encapsulated but not nonencapsulated Streptococcus pneumoniae. PLoS One 12, e0179159, doi:10.1371/journal.pone.0179159 (2017).

20. Kurihara, S. \& Suzuki, H. in Polyamines: A Universal Molecular Nexus for Growth, Survival, and Specialized Metabolism (eds Tomonobu Kusano \& Hideyuki Suzuki) 171-178 (Springer Japan, 2015).

21. Gerlin, L., Baroukh, C. \& Genin, S. Polyamines: double agents in disease and plant immunity. Trends Plant Sci, doi:10.1016/j.tplants.2021.05.007 (2021).

22. O'Neill, E. M. et al. Phevamine A, a small molecule that suppresses plant immune responses. 115, E9514-E9522, doi:10.1073/pnas.1803779115 \%J Proceedings of the National Academy of Sciences (2018).

23. Lowe-Power, T. M. et al. Metabolomics of tomato xylem sap during bacterial wilt reveals Ralstonia solanacearum produces abundant putrescine, a metabolite that accelerates wilt disease. Environ Microbio/ 20, 1330-1349, doi:10.1111/1462-2920.14020 (2018). 
24. Vilas, J. M. et al. Modulation of plant and bacterial polyamine metabolism during the compatible interaction between tomato and Pseudomonas syringae. J Plant Physio/ 231, 281-290, doi:10.1016/j.jplph.2018.09.014 (2018).

25. Shi, Z. et al. Putrescine Is an Intraspecies and Interkingdom Cell-Cell Communication Signal Modulating the Virulence of Dickeya zeae. 10, doi:10.3389/fmicb.2019.01950 (2019).

26. Das, K. C. \& Misra, H. P. Hydroxyl radical scavenging and singlet oxygen quenching properties of polyamines. Molecular and cellular biochemistry 262, 127-133, doi:10.1023/b:mcbi.0000038227.91813.79 (2004).

27. Tang, W. \& Newton, R. J. Polyamines reduce salt-induced oxidative damage by increasing the activities of antioxidant enzymes and decreasing lipid peroxidation in Virginia pine. Plant Growth Regulation 46, 31-43, doi:10.1007/s10725-005-6395-0 (2005).

28. Nayyar, H. \& Chander, S. Protective Effects of Polyamines against Oxidative Stress Induced by Water and Cold Stress in Chickpea. 190, 355-365, doi:https://doi.org/10.1111/j.1439-037X.2004.00106.x (2004).

29. Bors, W., Langebartels, C., Michel, C. \& Sandermann, H. Polyamines as radical scavengers and protectants against ozone damage. Phytochemistry 28, 1589-1595, doi:https://doi.org/10.1016/S0031-9422(00)97805-1 (1989).

30. Caldas, J. \& Vinga, S. Global Meta-Analysis of Transcriptomics Studies. PLOS ONE 9, e89318, doi:10.1371/journal.pone.0089318 (2014).

31. Xin, X. F., Kvitko, B. \& He, S. Y. Pseudomonas syringae: what it takes to be a pathogen. Nat Rev Microbiol 16, 316-328, doi:10.1038/nrmicro.2018.17 (2018).

32. Arnold, D. L. \& Preston, G. M. Pseudomonas syringae: enterprising epiphyte and stealthy parasite. Microbiology, doi:10.1099/mic.0.000715 (2018).

33. Rico, A. \& Preston, G. M. Pseudomonas syringae pv. tomato DC3000 uses constitutive and apoplastinduced nutrient assimilation pathways to catabolize nutrients that are abundant in the tomato apoplast. Mol Plant Microbe Interact 21, 269-282, doi:10.1094/MPMI-21-2-0269 (2008).

34. Xie, Y., Shao, X. \& Deng, X. Regulation of type III secretion system in Pseudomonas syringae. Environ Microbio/ 21, 4465-4477, doi:10.1111/1462-2920.14779 (2019).

35. Vandelle, E. et al. Transcriptional Profiling of Three Pseudomonas syringae pv. actinidiae Biovars Reveals Different Responses to Apoplast-Like Conditions Related to Strain Virulence on the Host. Mol Plant Microbe Interact 34, 376-396, doi:10.1094/mpmi-09-20-0248-r (2021).

36. Nobori, T. et al. Transcriptome landscape of a bacterial pathogen under plant immunity. 115, E3055e3064, doi:10.1073/pnas.1800529115 (2018).

37. Lovelace, A. H., Smith, A. \& Kvitko, B. H. Pattern-Triggered Immunity Alters the Transcriptional Regulation of Virulence-Associated Genes and Induces the Sulfur Starvation Response in Pseudomonas syringae pv. tomato DC3000. Mol Plant Microbe Interact 31, 750-765, doi:10.1094/mpmi-01-18-0008-r (2018). 
38. Nobori, T. et al. Multidimensional gene regulatory landscape of a bacterial pathogen in plants. Nature Plants 6, 883-896, doi:10.1038/s41477-020-0690-7 (2020).

39. Yu, X. et al. Transcriptional responses of Pseudomonas syringae to growth in epiphytic versus apoplastic leaf sites. Proc Natl Acad Sci U S A 110, E425-434, doi:10.1073/pnas.1221892110 (2013).

40. McAtee, P. A. et al. Re-programming of Pseudomonas syringae pv. actinidiae gene expression during early stages of infection of kiwifruit. BMC Genomics 19, 822, doi:10.1186/s12864-018-5197-5 (2018).

41. Helmann, T. C., Deutschbauer, A. M. \& Lindow, S. E. Genome-wide identification of Pseudomonas syringae genes required for fitness during colonization of the leaf surface and apoplast. Proc Natl Acad Sci U S A 116, 18900-18910, doi:10.1073/pnas.1908858116 (2019).

42. Helmann, T. C., Deutschbauer, A. M. \& Lindow, S. E. Distinctiveness of genes contributing to growth of $<$ em>Pseudomonas syringae</em> in diverse host plant species. 2020.2007.2028.216440, doi:10.1101/2020.07.28.216440 \%J bioRxiv (2020).

43. Nishad, R., Ahmed, T., Rahman, V. J. \& Kareem, A. Modulation of Plant Defense System in Response to Microbial Interactions. 11, doi:10.3389/fmicb.2020.01298 (2020).

44. Macho, A. P. Subversion of plant cellular functions by bacterial type-IIl effectors: beyond suppression of immunity. New Phyto/ 210, 51-57, doi:10.1111/nph.13605 (2016).

45. Lolle, S., Stevens, D. \& Coaker, G. Plant NLR-triggered immunity: from receptor activation to downstream signaling. Current opinion in immunology 62, 99-105, doi:10.1016/j.coi.2019.12.007 (2020).

46. Akhova, A. V. \& Tkachenko, A. G. Multifaceted role of polyamines in bacterial adaptation to antibioticmediated oxidative stress. Korean J. Microbiol. 56, 103-110, doi:10.7845/kjm.2020.0013 (2020).

47. Tkachenko, A. G., Akhova, A. V., Shumkov, M. S. \& Nesterova, L. Y. Polyamines reduce oxidative stress in Escherichia coli cells exposed to bactericidal antibiotics. Res Microbiol 163, 83-91, doi:10.1016/j.resmic.2011.10.009 (2012).

48. Chattopadhyay, M. K., Tabor, C. W. \& Tabor, H. Polyamines protect Escherichia coli cells from the toxic effect of oxygen. Proc Natl Acad Sci U S A 100, 2261-2265, doi:10.1073/pnas.2627990100 (2003).

49. Felix, J. et al. Structural and functional analysis of the Francisella lysine decarboxylase as a key actor in oxidative stress resistance. Sci Rep 11, 972, doi:10.1038/s41598-020-79611-5 (2021).

50. Jung, I. L. \& Kim, I. G. Transcription of ahpC, katG, and katE genes in Escherichia coli is regulated by polyamines: polyamine-deficient mutant sensitive to $\mathrm{H} 2 \mathrm{O} 2$-induced oxidative damage. Biochemical and Biophysical Research Communications 301, 915-922, doi:https://doi.org/10.1016/S0006291X(03)00064-0 (2003).

51. Guo, M., Block, A., Bryan, C. D., Becker, D. F. \& Alfano, J. R. \&lt;span class=\&quot;named-content genus-species\&quot; id=\&quot;named-content-1\&quot;\&gt;Pseudomonas syringae\&lt;/span\&gt; Catalases Are Collectively Required for Plant Pathogenesis. Journal of Bacteriology 194, 5054, doi:10.1128/JB.00999-12 (2012). 
52. O'Leary, B. M. et al. Early changes in apoplast composition associated with defence and disease in interactions between Phaseolus vulgaris and the halo blight pathogen Pseudomonas syringae Pv. phaseolicola. Plant Cell Environ 39, 2172-2184, doi:10.1111/pce.12770 (2016).

53. Winther, K. S., Sørensen, M. A. \& Svenningsen, S. L. Polyamines are Required for tRNA Anticodon Modification in Escherichia coli. J Mol Biol 433, 167073, doi:10.1016/j.jmb.2021.167073 (2021).

54. Liu, Z., Hossain, S. S., Morales Moreira, Z. \& Haney, C. H. Putrescine and its metabolic precursor arginine promote biofilm and c-di-GMP synthesis in Pseudomonas aeruginosa. J Bacteriol, Jb0029721, doi:10.1128/jb.00297-21 (2021).

55. Patel, C. N. et al. Polyamines are essential for the formation of plague biofilm. J Bacterio/ 188, 23552363, doi:188/7/2355 [pii] 10.1128/JB.188.7.2355-2363.2006 (2006).

56. El-Halfawy, O. M. \& Valvano, M. A. Putrescine Reduces Antibiotic-Induced Oxidative Stress as a Mechanism of Modulation of Antibiotic Resistance in Burkholderia cenocepacia. Antimicrobial Agents and Chemotherapy 58, 4162-4171, doi:10.1128/aac.02649-14 (2014).

57. Jelsbak, L., Thomsen, L. E., Wallrodt, I., Jensen, P. R. \& Olsen, J. E. Polyamines Are Required for Virulence in Salmonella enterica Serovar Typhimurium. PLOS ONE 7, e36149, doi:10.1371/journal.pone.0036149 (2012).

58. Chatnaparat, T., Prathuangwong, S. \& Lindow, S. E. Global Pattern of Gene Expression of Xanthomonas axonopodis pv. glycines Within Soybean Leaves. Mol Plant Microbe Interact 29, 508522, doi:10.1094/mpmi-01-16-0007-r (2016).

59. Lee, S. E. et al. Global Transcriptome Profiling of Xanthomonas oryzae pv. oryzae under in planta Growth and in vitro Culture Conditions. Plant Pathol J 33, 458-466, doi:10.5423/PPJ.OA.04.2017.0076 (2017).

60. Ward, N. \& Moreno-Hagelsieb, G. Quickly Finding Orthologs as Reciprocal Best Hits with BLAT, LAST, and UBLAST: How Much Do We Miss? PLOS ONE 9, e101850, doi:10.1371/journal.pone.0101850 (2014).

61. Sharma, R., Singh, G., Bhattacharya, S. \& Singh, A. Comparative transcriptome meta-analysis of Arabidopsis thaliana under drought and cold stress. PLOS ONE 13, e0203266, doi:10.1371/journal.pone.0203266 (2018).

62. Killcoyne, S., Carter, G. W., Smith, J. \& Boyle, J. Cytoscape: a community-based framework for network modeling. Methods Mol Bio/ 563, 219-239, doi:10.1007/978-1-60761-175-2_12 (2009).

63. Maere, S., Heymans, K. \& Kuiper, M. BiNGO: a Cytoscape plugin to assess overrepresentation of gene ontology categories in biological networks. Bioinformatics 21, 3448-3449, doi:10.1093/bioinformatics/bti551 (2005).

64. Bojanovič, K., D'Arrigo, I. \& Long, K. S. Global Transcriptional Responses to Osmotic, Oxidative, and Imipenem Stress Conditions in Pseudomonas putida. Appl Environ Microbio/ 83, doi:10.1128/aem.03236-16 (2017).

65. Jozefczuk, S. et al. Metabolomic and transcriptomic stress response of Escherichia coli. Molecular systems biology 6, 364, doi:10.1038/msb.2010.18 (2010). 
66. Chang, W., Small, D. A., Toghrol, F. \& Bentley, W. E. Microarray analysis of Pseudomonas aeruginosa reveals induction of pyocin genes in response to hydrogen peroxide. BMC Genomics 6, 115, doi:10.1186/1471-2164-6-115 (2005).

67. Liu, X., Omar, M., Abrahante, J. E., Nagaraja, K. V. \& Vidovic, S. Insights into the Oxidative Stress Response of Salmonella enterica serovar Enteritidis Revealed by the Next Generation Sequencing Approach. 9, 849 (2020).

\section{Figures}

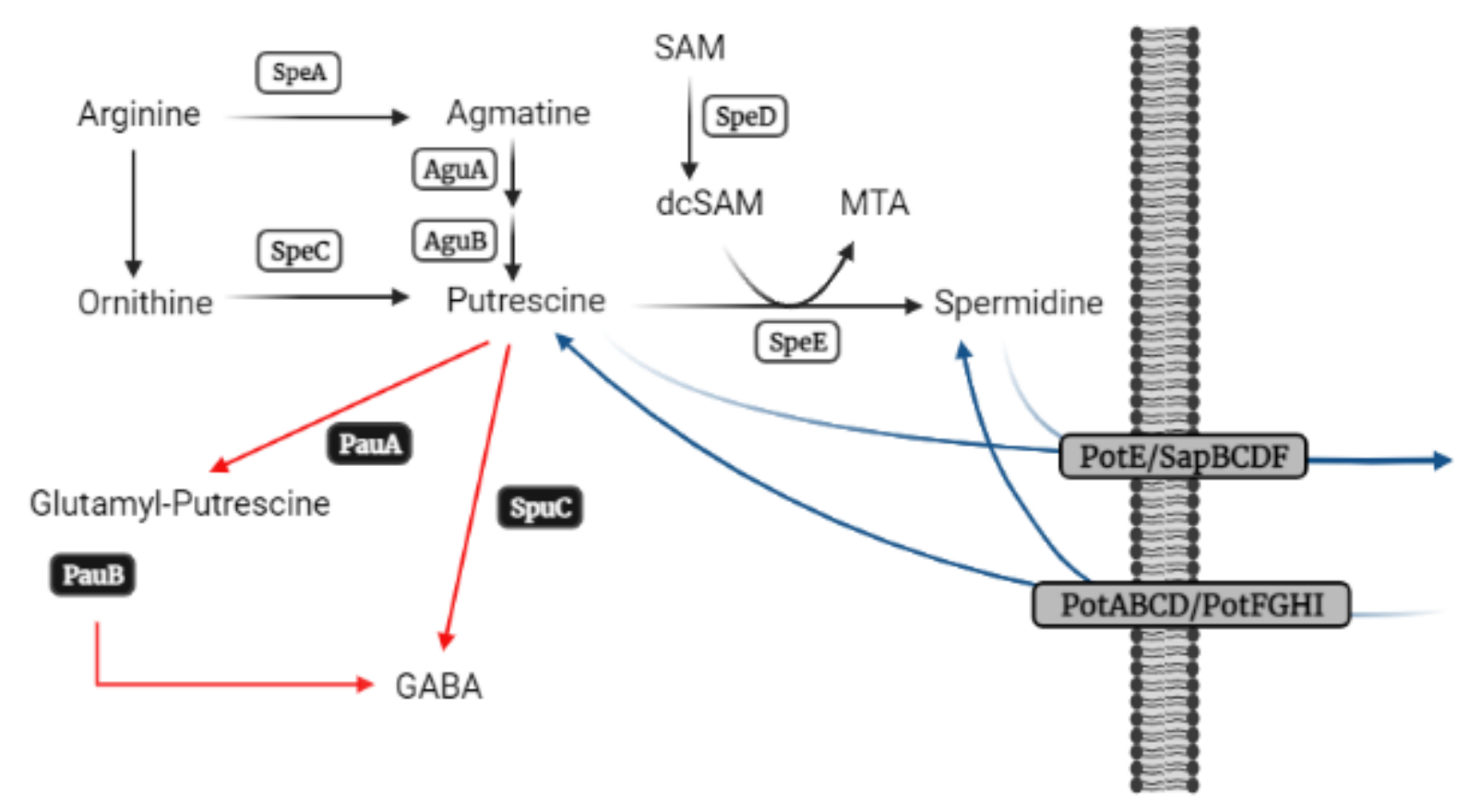

Figure 1

Schematic representation of polyamine metabolism pathways. Biosynthetic enzymes are represented in white rectangles, while black and grey rectangles depict the enzymes involved in polyamine catabolism and transport, respectively. Adapted from Schneider and Wendisch ${ }^{13}$ and created with BioRender.com 


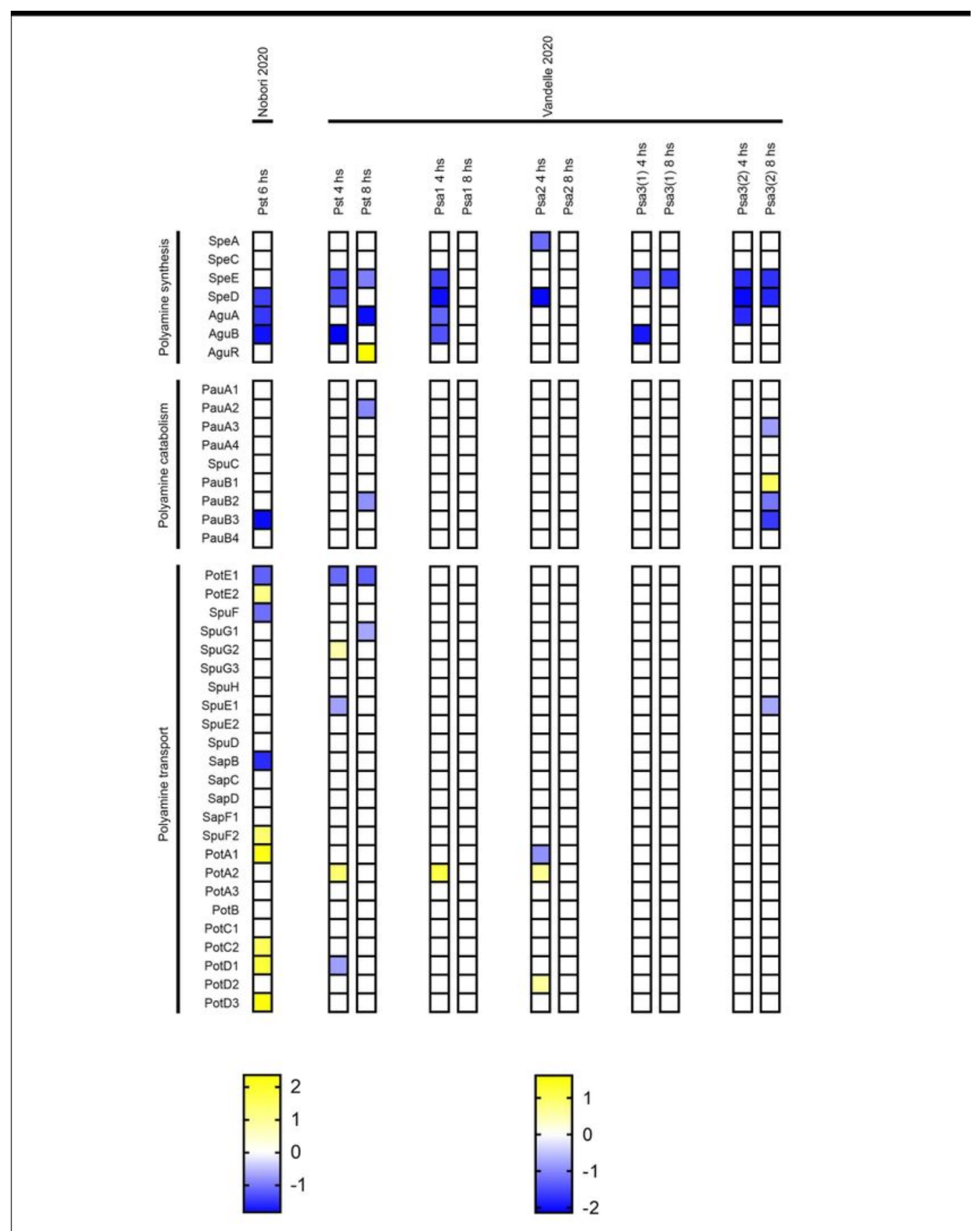

Figure 2

Heatmap representing polyamine metabolism gene expression levels in Pseudomonas syringae during growth in apoplastic-like conditions compared to rich media $\left(\left|\log _{2} \mathrm{FC}\right|>0.5, \mathrm{p}\right.$ adj<0.05). 
A

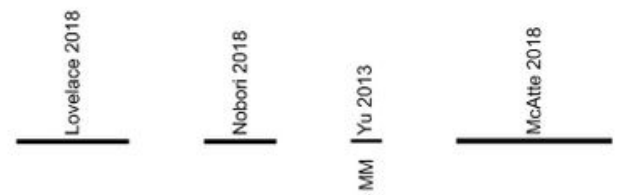

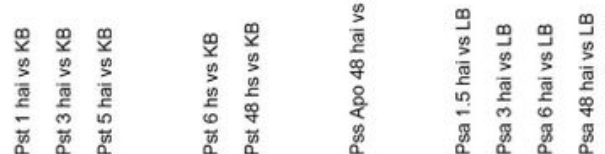
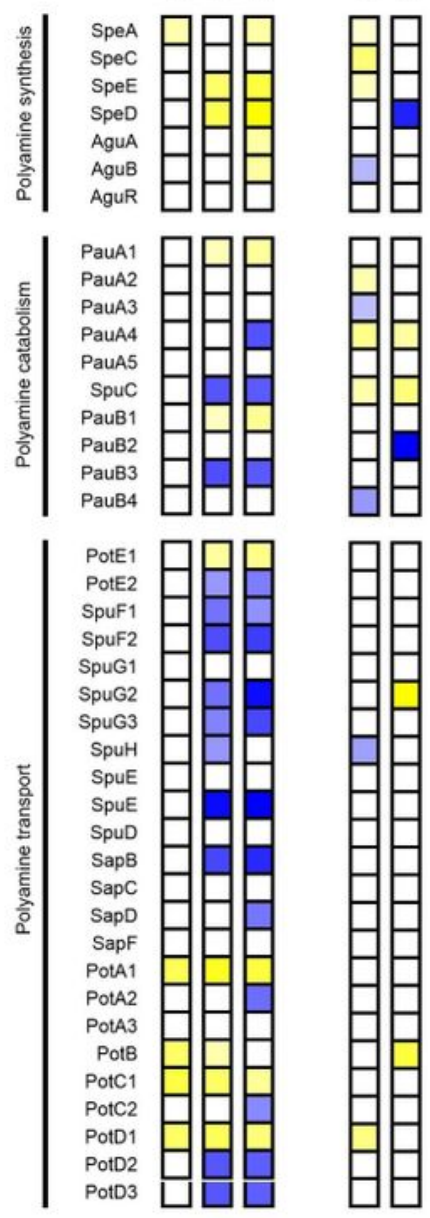

B
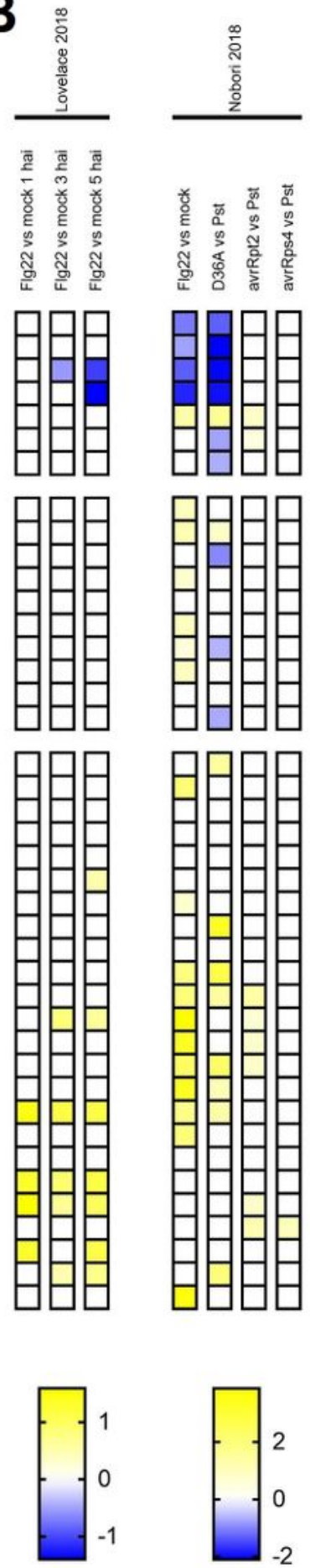

Figure 3

Polyamine metabolism gene expression in $P$. syringae during growth in plant tissues. Heatmap representing polyamine metabolism gene expression levels in $P$. syringae during $\mathbf{A}$ ) plant invasion in comparison to basal conditions and B) in Flg22-treated plants compared to mock, as well as non-virulent strains compared to WT Pst $\left(\left|\log _{2} \mathrm{FC}\right|>0.5, \mathrm{p}\right.$-adj< $\left.<0.05\right)$ 
Figure 4
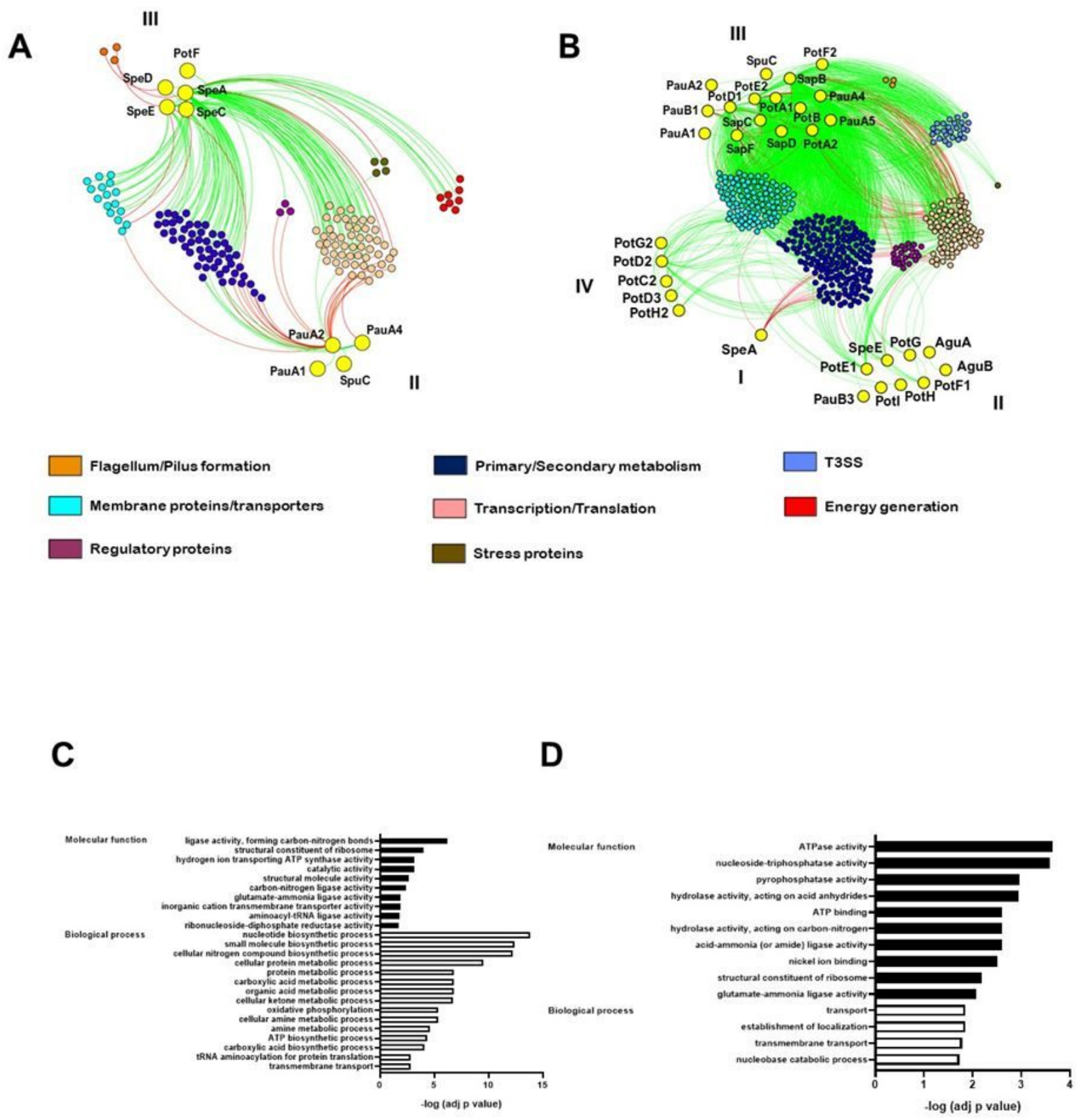

Figure 4

mRNA co-expression networks in $P$. syringae using the datasets from Nobori et al $(2018,2020)(A)$ and $Y u$ et al (2013) (B). Red and green edges denote negative and positive correlations, respectively, for highly correlated genes ( $R 2>0.75$ for Nobori et al, 2018, 2020; and $R 2>0.99$ for Yu et al, 2013)). Functions are color-coded. Pearson's correlation coefficients were used. Bar graphs in C) and D) depict GO-enrichment analysis of the genes included in A and B, respectively. 


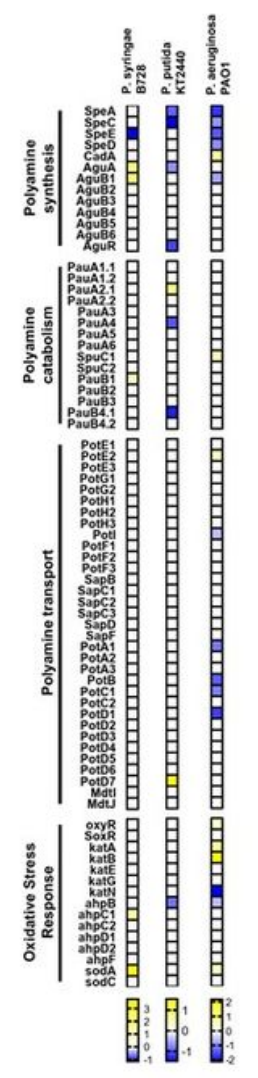

Figure 5

Heatmap showing polyamine metabolism and oxidative stress gene expression in Pseudomonas species during exposure to $\mathrm{H}_{2} \mathrm{O}_{2}$ in comparison to basal conditions $\left(\| \log _{2} \mathrm{FC} \mid>0.5\right.$, p adj<0.05). 


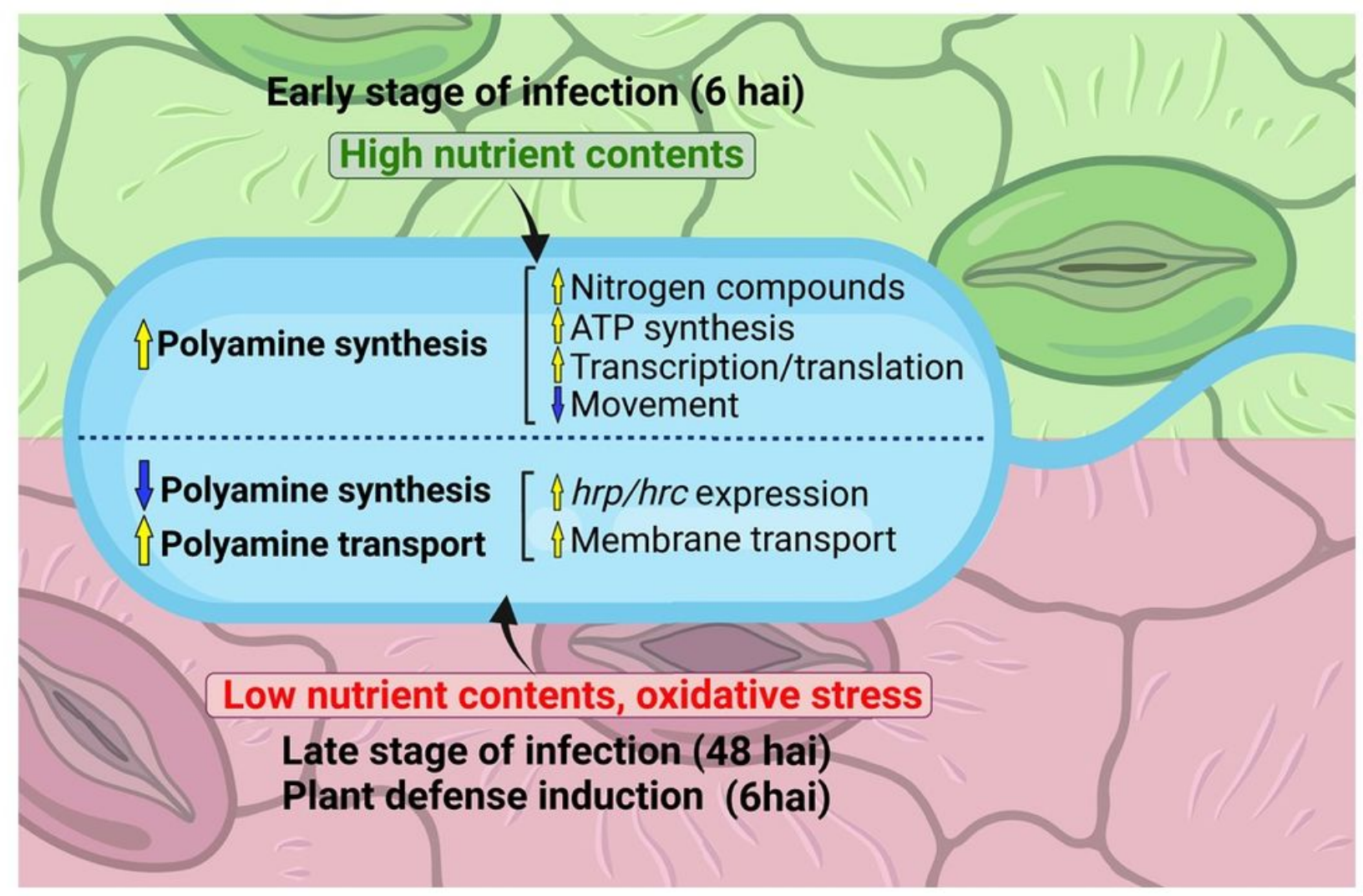

Figure 6

Legend not included with this version

\section{Supplementary Files}

This is a list of supplementary files associated with this preprint. Click to download.

- FigureS1.pptx

- Figures2.jpg

- TableS1.docx 